\title{
Voice Parameter Analysis for Early Detection of Autism in Children
}

\author{
Anasuodei Moko \\ Department of Computer Science and Informatics \\ Federal University Otuoke \\ Bayelsa State, Nigeria
}

\author{
Ledisi G. Kabari \\ Department of Computer Science \\ Ignatius Ajuru University of Education \\ Rivers State, Nigeria
}

\begin{abstract}
Autism Spectrum Disorder (ASD) is categorized by social, behavioral and must crucially communication deficiencies. Early detection is necessary for an improved positive result in therapy. This paper proposes a voice parameter analysis for the early detection of ASD in children. The Study used MATLAB programming software to display the signal analysis of an obtained voice data from an autistic and a normal child. The key experimental result shows a major difference in both voices in the time-domain, frequency, and spectrogram signal view. It is seen that the power spectrum (dB) set off with a high pitch level, at $-50 \mathrm{db}$ for the autistic child, while the normal child is slightly below but closer to $60 \mathrm{db}$ and it remained relatively stable for an autistic child along the normalized frequency within $0.58 \mathrm{hz}-1.0 \mathrm{hz}$. The paper thus recommends early voice screening of Autism Spectrum Disorder (ASD) as a significant first step to identify children at risk of the disease development and will need additional evaluation, intervention, and services.
\end{abstract}

\section{General Terms}

Digital Signal Processing

\section{Keywords}

Signal, voice, autism spectrum disorder, analysis, parameters

\section{INTRODUCTION}

The need for early detection of autism spectrum disorder in children is of uttermost importance in that its early identification is a key step in considering the risk of developing the disorder which requires early assessment, intervention, and optimal assessment. Voice medium is known as the most natural form of communication and as such an early voice screening of autism spectrum disorder (ASD) is a significant first step to identify children at risk of disease development and will need additional evaluation, intervention, and services. Recent years has recorded outstanding research in identifying the behavior and biological markers to help detect the signs of Autism Spectrum Conditions (ACD).[2] Autism is a spectrum of closely related disorders, not a particular disorder but is filled with a joint core of symptoms.[7] disclosed that the current study and estimation depicts those one out of 86 children are currently suffering autism spectrum diseases and neurodevelopmental disorders (NDDs), a figure that is growing at an alarming rate. Around ASD in the areas of diagnostic, therapy, and education many studies and practices are reported in current scientific literature. In support of compartmental observation, objective data and measurement about behavioral atopies that are distinctive to these disorders are becoming more and more important. Due to a combination of genetics, biology and environmental conditions, the brain develops rapidly during the first years of life alongside unusual development, all combined with adaptations that are caused by the typical interactions between the child in developing situations and his/her environment. This view suggests that the major neural disabilities leading to ASD may be transitory and therefore difficult to detect after a critical period of development. [1]

Autism Spectrum disorder comes with anxiety, depression, and obsessive-compulsive disorder (OCD), feeding issues, extremely restricted food habits that are often challenging and problem communicating with others of which an early detection will help in tackling and effectively manage these associated problems. [8]

The paper proposes an early detection of Autism Spectrum disorder in children using voice parameter analysis. This can be done by obtaining and analyzing the voice data of the child using MATLAB and compare normal child's voice and autistic child's voice using time-domain signal, frequency spectrum view of signals and spectrogram signal view in MATLAB

\section{REVIEW OF RELATED WORKS}

A study by undergoing a clarification of the characteristics of auditory information processing mechanism in 12 individuals with autism spectrum disorder carried out by [2], investigated 12 individuals (mean age $=11.83$ years) and 12 matched typical development $(\mathrm{TD})$ controls (mean age $=11.58$ years) the judgement was on the emotional valence of vocal stimuli with happy, angry, or neutral expressions, to detect the best correspondence to the semantics of vocal stimuli. The results show that importance of emotion conveyed verbally during conversations with individuals with ASD.

Certain commonly used ASD specific screening instruments and focused on the autism identification of young children with ASD utilizing an early childhood autism detection (ADEC), an ASD-specific screening tool developed in Australia as reviewed. [11] The ADEC was developed to serve as a timely, time-effective for children under the age of three, which is easy to administer, and suitable for those with little training and screening tools. The Study further reviewed key findings of an ADEC and some commonly used ASD specific screening tools, ADEC is a sound and efficient ASD screening tool, which is suitable for young children aged 1236 months. An online database of Psych-Information and Google Scholar was simultaneously searched for entries with any combination of (a) autism screening and (b) early childhood autism detection. The results of four studies indicate the sensitivity and specificity of ASD screening with $\mathrm{ADEC}$ in children under three years of age. ADEC is like some usual screening tools for ASD.

While exploring a feature representation learnt using a deep 
generative adversarial network (GAN) [2] classified the speech of children with developmental disorders. The study carried a comparative evaluation with another different acoustic feature. Results presented that GAN based methods show a competitive performance when compared to the conventional paradigm in terms of unweighted average recall metric.

A study on voice parameter analysis for detection of autism spectrum disorder used the MATLAB program to identify a patient with autism symptoms. The paper recorded five (5) voice samples and experimented on the voices using the cross-correlation technique to match them with recorded nonautistic voice. [6]

ASD issues commence in childhood and keep going into adolescence and adulthood. The paper proposed the possibility of using Naïve Bayes, Support Vector Machine, Logistic regression, KNN, Neural Network and Convolutional Neural Network for analyzing and predicting Autism Spectrum problem in a child, adolescents, and Adults. Three non-clinical datasets were evaluated which resulted in 292 instances and 21 attributes, second had 704 instances and 21 attributes, the third 104 instances and 21 attributes for Adult and Adolescents. [9] Results recorded after applying various machine learning techniques and handling missing values suggest that CNN based prediction models were better on all datasets with an accuracy of $99.5 \%, 99.3 \%$ and $96.8 \%$ of screening data.

To conduct a test on the relationships between vocal emotion and vocal pitch perception in adults with high autism spectrum disorder (ASD) and adults' pairs with typical development. [10] Results from the study indicated that problems of vocal emotional awareness in ASD may be based not only on problems with complex social tasks but also on problems with processing of fundamental sensory functions such as vocal pitch.

In a cry-based screening approach for children with autism spectrum disorder (ASD) opinedan early as well as automatic screening for children with autism spectrum disorder (ASD).[4] Recognized that ASD-specific characteristics in all children with ASD and collected from each child are not necessarily observed. the study from its observation proposed a new approach to properly classify, identify these characteristics and their respective instances. A collection of data on children between 18 and 53 months were examined to test the proposed approach and it was recorded using highquality voice recording systems and typical smartphones at different places such as homes or childcare center. The approach trained a classifier, using data for 10 boys with ASD and 10 Typically Developed (TD) boys. Data of 14 boys and 7 girls with ASD and 14 TD boys and 7 TD girls were tested for the trained classifier. The sensitivity, specificity, and precision of the proposed approach for boys were $85.71 \%, 100 \%$, and $92.85 \%$, respectively. These measures were $71.42 \%, 100 \%$, and $85.71 \%$ for girls, respectively. The proposed method was shown to exceed the popular techniques of classification. In addition, better results than studies using voice functions for ASD diagnosis have been illustrated.

\section{METHODOLOGY}

Since this study is performed on children subjects, first, the parents of the participants were informed about the study and approval was given. The voice analysis is achieved with a comparison of a normal child and a child with ASD based on these parameters: frequency, pulse, amplitude, harmonic nature of the voice and pitch. In this research, the signal analyzer application was adopted in MATLAB. The Signal Analyzer application is an interactive tool for time-domain, frequency-domain and time-frequency-domain visualization, preprocessing, measurement, analysis, and comparisonof signal.

\section{RESULTS AND DISCUSSION}

The results of the analysis of a normal child and an autistic child are shown in Figure 1- 4 with a sample frequency at $48000 \mathrm{~Hz}$. The voice signal of an autistic child analyzed,(See Figure 1) while(Figure 2) shows the voice signal of a normal child and (Figure 3)displays the autistic and normal child signal in the time-domain, frequency, and spectrogram signal view and Figure 4 shows the combination normal and autistic signal in time-domain and frequency view.

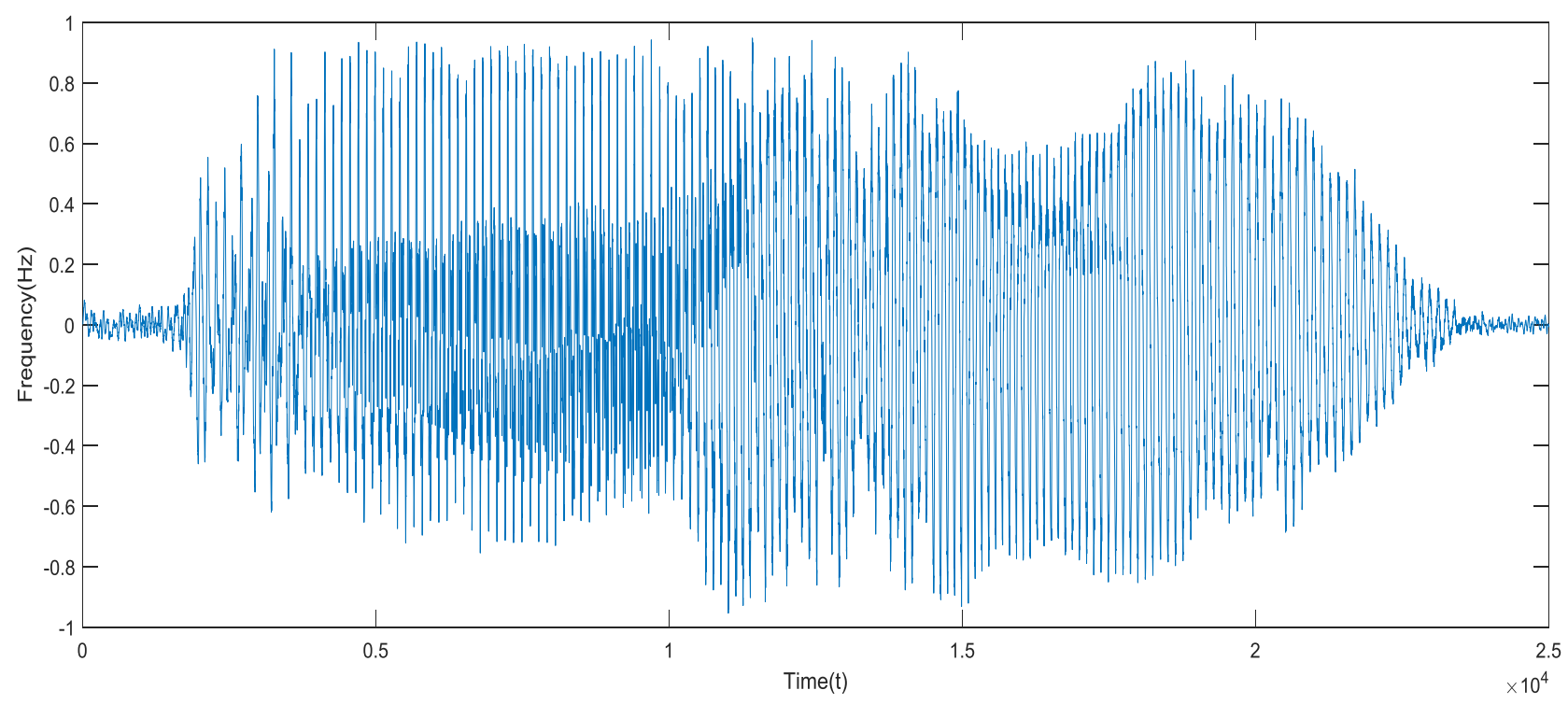

Fig 1: Voice of an Autistic Child 


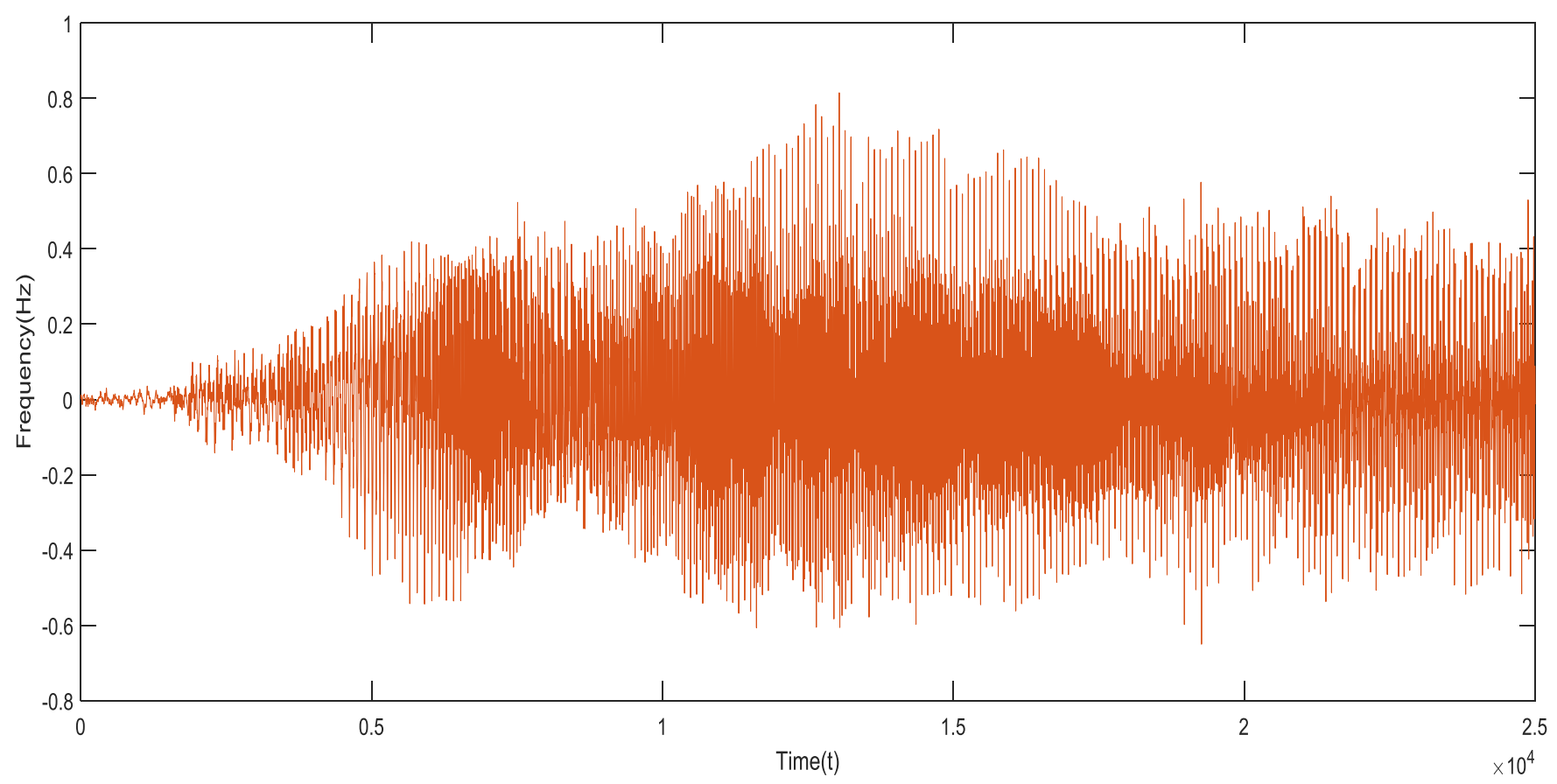

Fig 2: Voice of a Normal Child
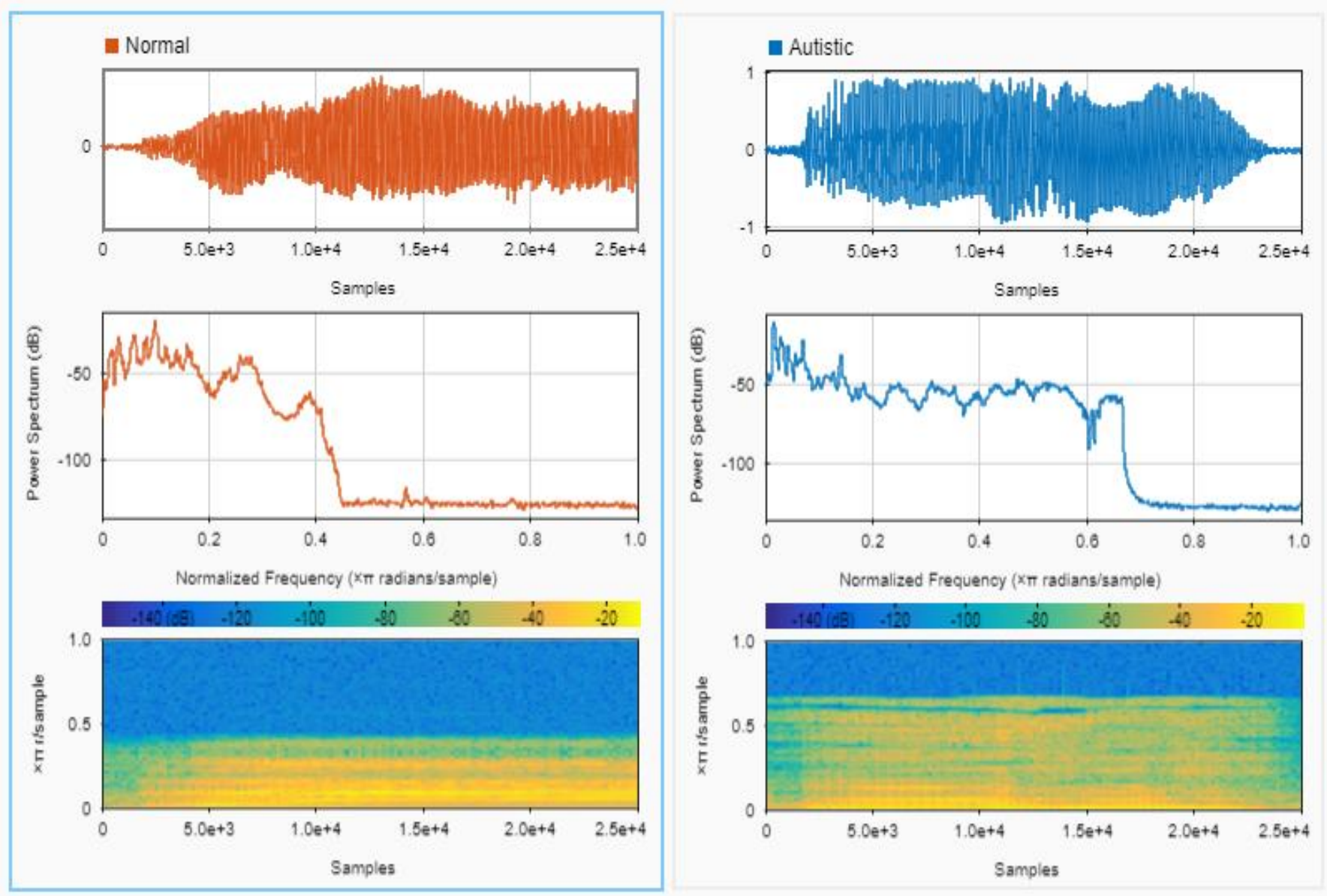

Fig 3.Comparison Normal and Autistic Voice Signal Analysison time-domain, frequency, and spectrogram signal view 


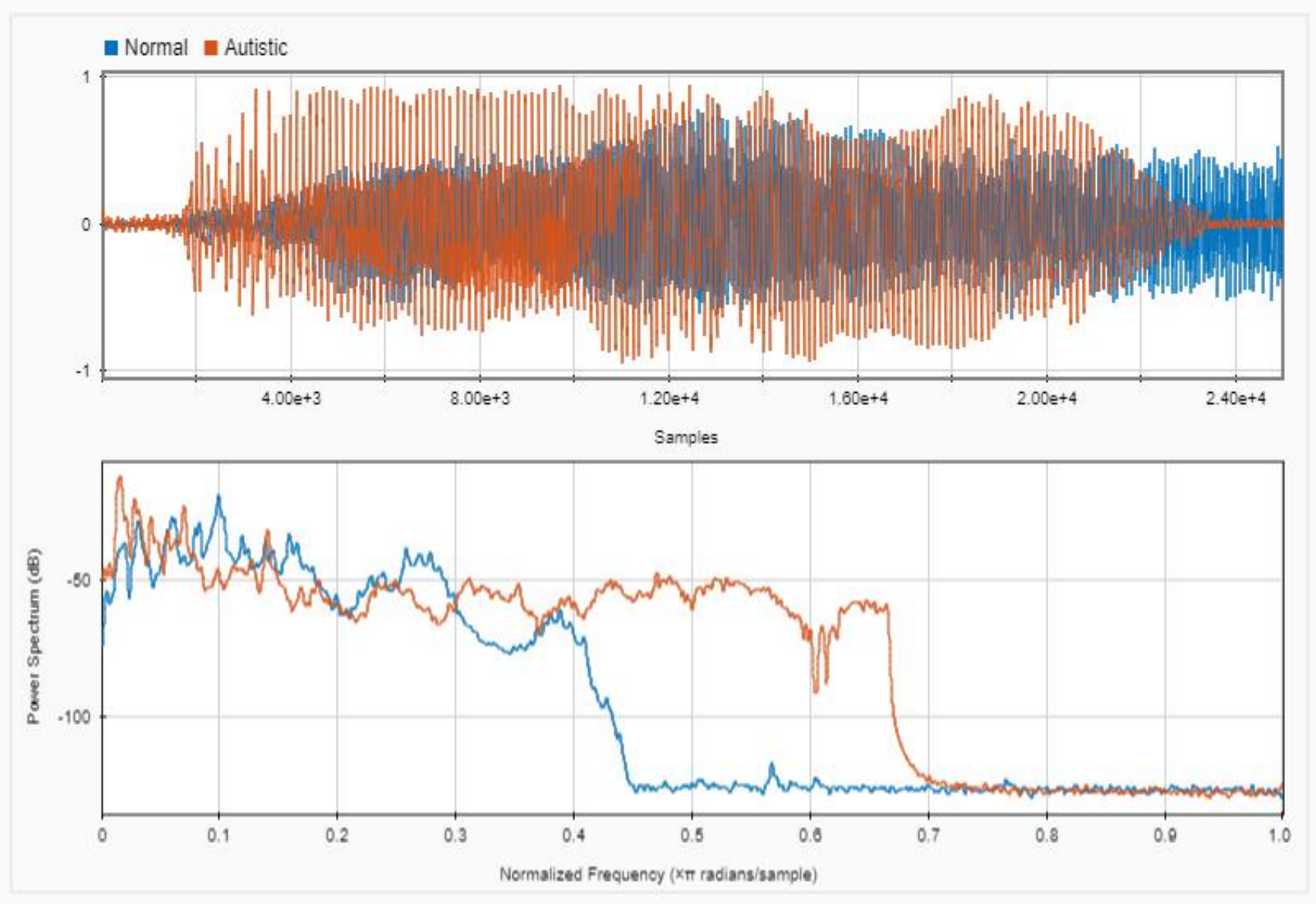

Fig 4: Combination of Normal and Autistic Voice Signal on time-domain and frequency view

The signal level is higher from the onset for an autistic child starting from zero and improved over a period with a higher pitch level as compared to that of a normal child (see Figure 3 ). In a normal child, the frequency against time plots, it is seen that the frequency level began from the onset of the child, meaning all factors causing autism has been addressed.The second result where both voices placed together (see Figure 4)which shows the compared results for a normal and an autistic child. It is seen that the power spectrum (dB) set off with a high pitch level, at $-50 \mathrm{db}$ for the autistic child, while the normal child is slightly higher than $-70 \mathrm{db}$ and it remained relatively stable for an autistic child along the normalized frequency within $0.7 \mathrm{hz}$ to $1.0 \mathrm{hz}$. Hence, in this investigation we have been able to show the comparison deduced from the analysis of normal and autistic voice data. The problem of autism spectrum disorder comes with anxiety, depression, and obsessive-compulsive disorder (OCD), feeding issues, extremely restricted food habits that are often challenging and problem communicating with others. Hence there is need to watch those contributing facts in trying to minimize autism issues in the world.

Table 1. Voice Analysisfor Autistic and NormalChild

\begin{tabular}{|c|c|c|}
\hline Parameters & Autistic Child & Normal Child \\
\hline Voice Time & 2.5 Seconds & 2.5 Seconds \\
\hline $\begin{array}{c}\text { Normalized } \\
\text { Frequency }\end{array}$ & 0.7 radius/sample & 0.46 radius/sample \\
\hline Amplitude & $0.1809(\mathrm{~m})$ & $0.096(\mathrm{~m})$ \\
\hline Pitch level & $-50 \mathrm{~dB}$ & $-74.3254 \mathrm{~dB}$ \\
\hline
\end{tabular}

As seen in Table 1, the normalized frequency is a frequency which ranges from 0 to 1 expressed in units of cycles (sample) per radius is know as the standard frequency divided into $\mathrm{Hz}$ by the sample frequency of the signal (or the same units as your original frequency). So, your sample rate is a normalized frequency of 1 and 0.5 is the sample frequency of Nyquist a minimum sampling speed at which a signal can be evaluated without errors, which is half as high as the signal frequency. The pitch levelindicates the highest level of the voice while the amplitude is the space between the starting point and the peak of the voice.

\section{CONCLUSION}

Autism is a spectrum of closely related disorders, not a particular disorder but is filled with a joint core of symptoms. Early voice screening of autism spectrum disorder (ASD) is a significant first step to identify children at risk of disease development and will need additional evaluation, intervention, and services. One out of 86 children are currently suffering from autism spectrum diseases and neurodevelopmental disorders. This study has experimental showed through the voice analysis carried out using the signal analyzer tool in MATLAB the difference in the voice of a normal child and an autistic child.

\section{ACKNOWLEDGEMENT}

All gratitude goes to the parents of the autistic and normal child that provided the voices and gave their permission for the achievement of this research.

\section{REFERENCES}

[1] Bosl, W. J., Tager-Flusberg, H., \& Nelson, C. A. (2018). EEG Analytics for Early Detection of Autism Spectrum 
Disorder: A data-driven approach. Scientific Reports, 8.

[2] Deng, J., Cummins, N., Schmitt, M., Qian, K., Ringeval, F., \& Schuller, B. (2017). Speech-based Diagnosis of Autism Spectrum Condition by Generative Adversarial Network Representations. 7th International Digital Health Conference, 5, 53-57.

[3] Fusaroli, R., Anna Lambrechts, Dan Bang, M Bowler, D., \& B Gaigg, S. (n.d.). "Is Voice A Marker for Autism Spectrum Disorder? A Systematic Review and MetaAnalysis." 64.

[4] Khozaei, A., Moradi, H., Hosseini, R., Pouretemad, H., \&Eskandari, B. (2020). Early screening of autism spectrum disorder using cry features. PLOS ONE, 15(12).

[5] Matsumoto, K., Sugiyama, T., Saito, C., Kato, S., Kuriyama, K., Kanemoto, K., \& Nakamura, A. (2016). Behavioural Study on Emotional Voice Perception in Children with Autism Spectrum Disorder. Journal of Pediatric Neuropsychology, 2(3-4), 108-118.

[6] Nne R., S., \& Ledisi G., K. (2018). Voice Parameter Analysis for Detection of Autism Spectrum Disorder. Journal of Environmental Science, Computer Science and Engineering \& Technology, 9(1), 8.
[7] Orlandi_Silvia_Tesi.pdf. (n.d.). Retrieved February 13, 2021, from http://amsdottorato.unibo.it/7149/1/Orlandi_Silvia_Tesi. pdf

[8] Patten, E., Belardi, K., Baranek, G. T., Watson, L. R., Labban, J. D., \&Oller, D. K. (2014). Vocal patterns in infants with autism spectrum disorder: Canonical babbling status and vocalization frequency. Journal of Autism and Developmental Disorders, 44(10), 2413 2428.

[9] Raj, S., \& Masood, S. (2020). Analysis and Detection of Autism Spectrum Disorder Using Machine Learning Techniques. Procedia Computer Science, 167, 994-1004.

[10] Schelinski, S., \&Von Kriegstein, K. (2019). The Relation Between Vocal Pitch and Vocal Emotion Recognition Abilities in People with Autism Spectrum Disorder and Typical Development. Journal of Autism and Developmental Disorders, 49(1), 68-82.

[11] Young, R. L., \& Nah, Y. (2016). Examining Autism Detection in Early Childhood (ADEC) in the Early Identification of Young Children with Autism Spectrum Disorders (ASD). Australian Psychologist, 51(4), 261271. 